Corrected: Author Correction

\title{
Targeting vesicle size
}

\author{
An amphipathic peptide has been engineered and is capable of penetrating the blood-brain barrier as well as \\ possessing a potent antiviral activity against Zika and other mosquito-borne viruses.
}

Jing Zou and Pei-Yong Shi

$\mathrm{O}$ ne third of deaths from infectious diseases worldwide are of viral origins. Although hundreds of viruses are known to cause human diseases, the mainstay of antiviral research and development remains focused on direct antiviral agents (DAA) that inhibit specific viral proteins of individual viruses. This 'one lock, one key' approach has resulted in two major drawbacks of the current antiviral therapy: narrow spectrum of therapeutic coverage and emergence of drug resistance. These drawbacks are further inflamed by two daunting challenges. First, the development of a new drug is slow and expensive, taking at least ten years at an average cost of over US $\$ 2$ billion dollars ${ }^{1}$. Second, the nature of virus emergence is very unpredictable. The one lock, one key approach is impossible to provide a rapid countermeasure when responding to a global crisis of virus emergence, as evidenced during the recent epidemics of severe acute respiratory syndrome (SARS), Middle East respiratory syndrome (MERS), chikungunya, Ebola and Zika viruses. Thus, new antiviral approaches are urgently needed. Writing in Nature Materials, Joshua Jackman and colleagues ${ }^{2}$ report an antiviral approach, termed lipid envelope antiviral disruption (LEAD), for development of broad-spectrum antiviral therapy. The authors demonstrated that an amphipathic $\alpha$-helical peptide (AH-D), derived from the first 27 amino acids of hepatitis $\mathrm{C}$ virus (HCV) non-structural protein 5A (NS5A; Fig. 1a), has potent antiviral activities against Zika, dengue, yellow fever, Japanese encephalitis and chikungunya viruses, with $\mathrm{EC}_{50 \mathrm{~s}}$ (a concentration required to inhibit $50 \%$ of viral replication; $12-206 \mathrm{nM}$ ) and $\mathrm{CC}_{50}$ (a concentration required to cause $50 \%$ cytotoxicity; $63 \mu \mathrm{M})$. Remarkably, treatment of Zika virus-infected mice with this peptide, even when starting the therapy on day three post-infection, protected against disease and death without adverse effects.

The N-terminal region of $\mathrm{HCV}$ NS5A is known to associate with the endoplasmic reticulum membrane in infected cells. When studying the interaction of the $\mathrm{N}$-terminal $\alpha$-helix of this protein with lipid membranes, Cho et al. serendipitously discovered that the $\mathrm{AH}$ peptide could potently rupture liposomes ${ }^{3}$. Importantly, the AH peptide selectively ruptures liposomes with diameters of $<160 \mathrm{~nm}$. Such size-dependent liposome disruption is determined by the ability of the $\mathrm{AH}$ peptide to sense different degrees of membrane curvature ${ }^{4}$. Specifically, liposomes of $<160 \mathrm{~nm}$ have high membrane curvatures that are susceptible to $\mathrm{AH}$ peptide-mediated pore formation (Fig. 1b, top); once a critical density of pores is formed, membrane lysis occurs (Fig. 1b, bottom). In contrast, liposomes of $>160 \mathrm{~nm}$ have low membrane curvatures that are not susceptible to $\mathrm{AH}$ peptide-mediated pore formation, thus no lysis occurs ${ }^{5}$. In agreement with the liposome results, tested viruses prone to $\mathrm{AH}$ peptide inhibition fall within the disruptive range of liposome size: flaviviruses, 40-60 nm; chikungunya virus, 60-70 nm; bunyaviruses, 90-100 nm; and human immunodeficiency virus, $120 \mathrm{~nm}$. In contrast, viruses of larger sizes, such as vaccinia virus, $360 \times 270 \times 250 \mathrm{~nm}^{3}$, as well as cell membranes are less sensitive to the peptide inhibition ${ }^{2,6}$. These results suggest that the AH peptide may also inhibit other untested enveloped viruses, as long as their virions are $<160 \mathrm{~nm}$.

Besides AH peptide, Chisari and colleagues also found that C5A peptide, representing amino acids 3-20 of $\mathrm{HCV}$ NS5A, has a broad spectrum of antiviral activity $^{7,8}$. The potency of C5A peptide ( $\mathrm{EC}_{50 \mathrm{~s}} 0.6-4.5 \mu \mathrm{M}$ against different viruses) is much weaker than the current $\mathrm{AH}$ peptide, possibly due to the differences in peptide lengths and amino acid sequences. Indeed, mutagenesis analysis showed that the amphipathic $\alpha$-helical structure as well as the amino acid composition are critical for the peptide's antiviral activity ${ }^{7}$. Since chirality is not essential for antiviral activity, the current AH-D peptide was synthesized with $\mathrm{D}$-amino acids rather than L-amino acids that are more susceptible to proteolytic degradation 9 . The D-chirality improves the peptide's pharmacokinetic stability to a half-life time of $7 \mathrm{~h}$ in mice. In addition,
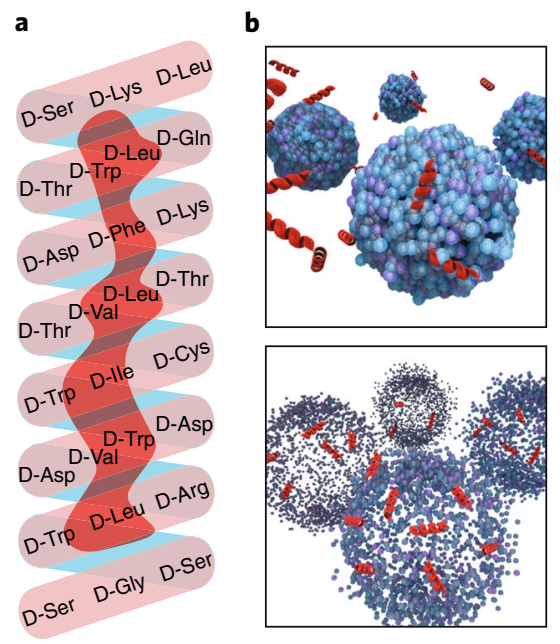

Fig. 1 | AH-D peptide and its membranedisruptive activity against liposomes. a, Helix net diagram of $\mathrm{AH}-\mathrm{D}$ peptide. The $\mathrm{N}$-terminus of the peptide starts at the bottom of the helix. b, Illustration of AH-D peptide (red) disrupting the membrane of liposomes (blue) with diameters of $<160 \mathrm{~nm}$.

the AH-D peptide can cross blood-brain barrier $^{2}$. This brain-penetrating property is ideal for potential treatment of neurotrophic viruses. Indeed, the improved potency, pharmacokinetics and brain-penetrating property of the AH-D peptide have translated into excellent efficacy in vivo. Treatment of Zika virus-infected mice with AH-D peptide reduced viral loads in blood and organs, as well as reduction in inflammatory cytokines, weight loss and deaths (Fig. 2). Specifically, treatment with AH-D peptide decreased the death rate from $100 \%$ to $17 \%$. The peptide's ability to access the brain significantly enhanced its antiviral activity in the central nervous system and protected the brain function of infected mice, as indicated by a normal level of myeloperoxidase ${ }^{2}$.

Targeting host factors essential for virus life cycle is a valid antiviral approach. This approach has led to the development of maraviroc, a clinically approved HIV drug that inhibits host protein CCR5, a co-receptor for HIV entry ${ }^{10}$. Compared 

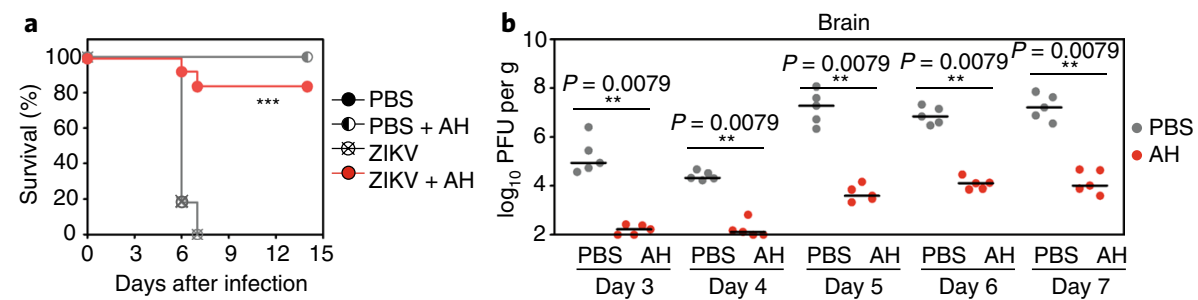

Fig. 2 | In vivo efficacy of AH-D peptide in mice. a,b, Type-I interferon receptor knockout mice (IFN- $\left.\alpha / \beta \mathrm{R}^{-/-}\right)$were intravenously infected with $4 \times 10^{3}$ plaque-forming units (PFU) of Zika virus (ZIKV). The infected mice were treated with $25 \mathrm{mg} \mathrm{kg}^{-1} \mathrm{AH}-\mathrm{D}$ peptide or phosphate-buffered saline (PBS) mock on days three to six post-infection. The mice were monitored for survival (a). The levels of infectious virus within the brain (b) were measured on days three to seven post-infection. The AH-D treatment significantly decreased viral loads in serum and various organs, leading to improved survival. Adapted from ref. ${ }^{2}$, Springer Nature Ltd.

with direct antiviral agents, host-targeting antiviral approach offers the advantage of higher barriers for resistance emergence, since viral mutations are less able to compensate for loss of essential host cofactors. In addition, because different viruses share common host factors/ pathways for viral replication, compounds targeting the shared host factors/pathways could have broad antiviral activities. For further development of the AH-D peptide, understanding the structure of how the peptide senses and disrupts the virions will help design future inhibitors. The ability to optimize and shorten the peptide, while keeping its potency, will reduce the drug cost. Most importantly, achieving no observed adverse effect level (NOAEL) in preclinical safety tests will advance the AH-D peptide to clinical trials. Overall, the current study represents a breakthrough in advancing the LEAD concept as a new class of broad-spectrum antiviral therapy.

\section{Jing Zou and Pei-Yong Shi*}

Department of Biochemistry and Molecular Biology, University of Texas Medical Branch, Galveston, TX, USA.

*e-mail:peshi@utmb.edu

Published online: 23 October 2018

https://doi.org/10.1038/s41563-018-0200-8
References

1. DiMasi, J. A., Grabowski, H. G. \& Hansen, R. W. J. Health Econ. 47, 20-33 (2016).

2. Jackman, J. A. et al. Nat. Mater. https://doi.org/10.1038/s41563018-0194-2 (2018).

3. Cho, N.-J., Cho, S.-J., Cheong, K. H., Glenn, J. S. \& Frank, C. W. J. Am. Chem. Soc. 129, 10050-10051 (2007).

4. Tabaei, S. R., Rabe, M., Zhdanov, V. P., Cho, N.-J. \& Höök, F. Nano Lett. 12, 5719-5725 (2012).

5. Jackman, J. A., Goh, H. Z., Zhdanov, V. P., Knoll, W. \& Cho, N.-J. J. Am. Chem. Soc. 138, 1406-1413 (2016).

6. Cho, N. J. et al. ACS Chem. Biol. 4, 1061-1067 (2009).

7. Cheng, G. et al. Proc. Natl Acad. Sci. USA 105, 3088-3093 (2008).

8. Bobardt, M. D. et al. Proc. Natl Acad. Sci. USA 105, $5525-5530$ (2008).

9. Garton, M. et al. Proc. Natl Acad. Sci. USA 115, 1505-1510 (2018).

10. Abel, S., Back, D. J. \& Vourvahis, M. Antivir. Ther. 14, 607-618 (2009).

\section{TWO-TERMINAL DEVICES}

\section{D materials-wrapped microparticles}

Colloidal microparticles, with polymer composites encapsulated within two separate 2D material sheets, are fabricated by autoperforation, which can carry chemical and electronic information with long-term instability in complex environments.

\section{Qiyuan He and Hua Zhang}

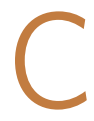
onforming materials to rigid substrates with Gaussian curvature can generate strain field and guide its crack propagation. This concept has been recently demonstrated at the macroscopic scale for controlling cracking morphology and producing desired shapes ${ }^{1}$. Now reporting in Nature Materials, Pingwei Liu and colleagues ${ }^{2}$ have successfully scaled down the concept to spontaneously assemble polymer composite ink and a pair of two-dimensional (2D) material sheets into functional microparticles, via guided cracking, termed autoperforation. It is a simple yet elegant nanofabrication technique that performs well with 2D materials such as graphene, transition metal dichalcogenides and hexagonal boron nitride, allowing a precise compositional or structural control of the exterior 2D material encapsulation and interior composite fillers, to achieve various functions for practical purpose.

A schematic of autoperforation process is shown in Fig.1a. Polymer discs with an appropriate aspect ratio of radius to height, the array of which can be fabricated by inkjet printing as shown in Fig. 1b, are sandwiched by two layers of $2 \mathrm{D}$ materials supported on poly(methyl methacrylate) (PMMA) films. During the lift-off process, 2D materials crack along the edges of the solid templates, forming a stable colloidal suspension of encapsulated microparticles. In this platform, different types of 2D materials can be chosen as top or bottom layers to have a hybrid encapsulation, while the polymer fillers can be composited with functional nanomaterials for different applications.

The flat surface and flexible nature of 2D materials render them perfect matrix to composite a variety of nanomaterials. For example, self-assembled transition metal dichalcogenide nanoscrolls have shown high loading amount of semiconducting or biological nanomaterials ${ }^{3}$. Methods of assembling 2D materials into microstructures ${ }^{4}$ to incorporate functional nanomaterials, such as warping ${ }^{5}$, scrolling ${ }^{3,6,7}$ and layer-by-layer assembly ${ }^{8}$, are widely applied in various fields. However, most reported techniques relied on bottom-up self-assembly processes, which lack the control on individual microstructures due to the inherent stochasticity of the process, with few exception of controlled top- 